\title{
The ATLAS Muon Trigger
}

\author{
Shota Hayashida ${ }^{1, *}$ on behalf of the ATLAS Collaboration \\ ${ }^{1}$ Nagoya University, Japan
}

\begin{abstract}
Events containing muons in the final state are important for many physics analyses performed by the ATLAS experiment at the Large Hadron Collider. To collect such events, an efficient and well-understood muon trigger is required. The ATLAS muon trigger consists of a hardware-based and a software-based subsystem. In order to cope with the high luminosity and pileup conditions in Run 2, several improvements have been implemented to suppress the trigger rate while maintaining a high efficiency. Recent improvements include addition of layers in the coincidence of the muon spectrometer and optimisation of a muon trigger isolation requirement, among others. An overview of the algorithms deployed by the ATLAS muon trigger and its performance in 2018 data taking is presented.
\end{abstract}

\section{Introduction}

The ATLAS experiment [1] installed at the Large Hadron Collider (LHC) started data taking of Run 2 in 2015 with a center of mass energy of $\sqrt{s}=13 \mathrm{TeV}$. The Run 2 data taking will continue until the end of 2018. The ATLAS trigger system is essential to efficiently select the events of high interest for physics analyses. Events containing muons in the final state are important for many analyses, such as searches for new particles and precision measurements of the Standard Model. An efficient muon trigger is vitally important to accumulate these events.

The ATLAS muon trigger in Run 2 is designed as a two-stage system that consists of a hardware-based trigger system (Level 1 muon trigger) and a software-based reconstruction system (High Level muon trigger). In order to cope with the high luminosity and pileup conditions in Run 2, several improvements have been implemented in the muon trigger to suppress the trigger rate while maintaining a high trigger efficiency.

\section{The ATLAS muon trigger}

The ATLAS muon trigger uses the information provided by the Muon Spectrometer (MS) and the Inner Detector (ID) of the ATLAS detector in order to select a high quality dataset of muons. The MS consists of four types of subdetectors with different purposes and three large air-core superconducting toroids as shown in Figure 1. Three layers of Resistive Plate Chambers (RPCs) in the central region $(|\eta|<1.05)$ and three layers of Thin Gap Chambers (TGCs) in the endcap regions $(1.05<|\eta|<2.4)$ provide fast reconstruction of muon candidates for the Level 1 muon trigger. ${ }^{1}$ Three or two layers of Monitored Drift Tube chambers (MDTs)

\footnotetext{
*e-mail: shota.hayashida@cern.ch

Copyright 2018 CERN for the benefit of the ATLAS Collaboration. CC-BY-4.0 licence

${ }^{1}$ The pseudorapidity is defined in terms of the polar angle $\theta$ as $\eta=-\ln \tan (\theta / 2)$
} 


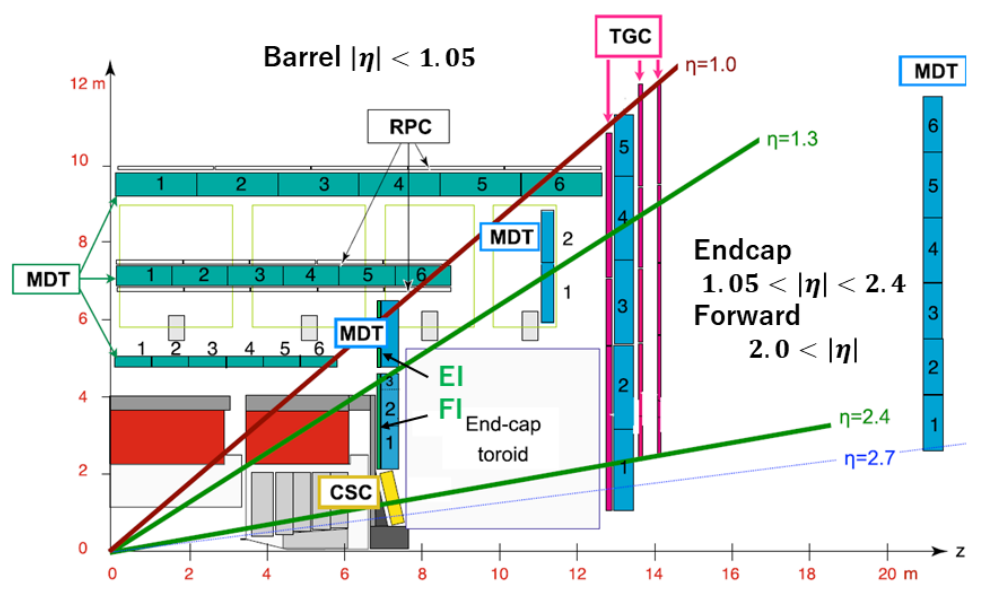

Figure 1. Schematic drawing of one quarter cross-section of the muon system of the ATLAS detector [2].

covering the central, the endcap and a part of the forward regions $(|\eta|<2.7)$ and one layer of Cathode Strip Chambers (CSCs) covering a part of the forward regions $(2.0<|\eta|<2.7)$ provide precise track information for the High Level muon trigger and offline muon reconstruction.

The ATLAS muon trigger selects the events including muon candidates with transverse momentum $p_{\mathrm{T}}$ greater than a predefined threshold. In Run 2, one of the primary high $p_{\mathrm{T}}$ triggers consists of a Level 1 muon trigger with a $20 \mathrm{GeV}$ threshold and a High Level muon trigger with a $26 \mathrm{GeV}$ threshold.

\section{Improvements of the Level 1 muon trigger for Run 2}

The Level 1 muon trigger requires spatial and temporal coincidence on the hits in the RPCs and TGCs. The muon $p_{\mathrm{T}}$ is estimated by using the degree of deviation from the hit pattern of an infinite momentum assumption [2]. In Run 1, the Level 1 muon trigger rates in the forward regions were polluted by low $p_{\mathrm{T}}$ charged particles, which were considered to be mostly protons originating from beam background.

To suppress such fake events, an additional coincidence of the TGCs has been implemented in Run 2. It is based on the small-wheel TGCs, the forward inner (FI) and the endcap inner (EI) chambers placed in front of the endcap toroidal magnet in the part of the endcap regions $(1.05<|\eta|<2.0)$ shown in Figure 1. Figure 2 shows the $\eta$ distributions of the Level 1 muon trigger for a $p_{\mathrm{T}}$ threshold of $20 \mathrm{GeV}$ (L1_MU20) with and without the additional coincidence. The additional coincidence reduces the trigger rate by about $20 \%$ with efficiency losses below $1 \%$. 


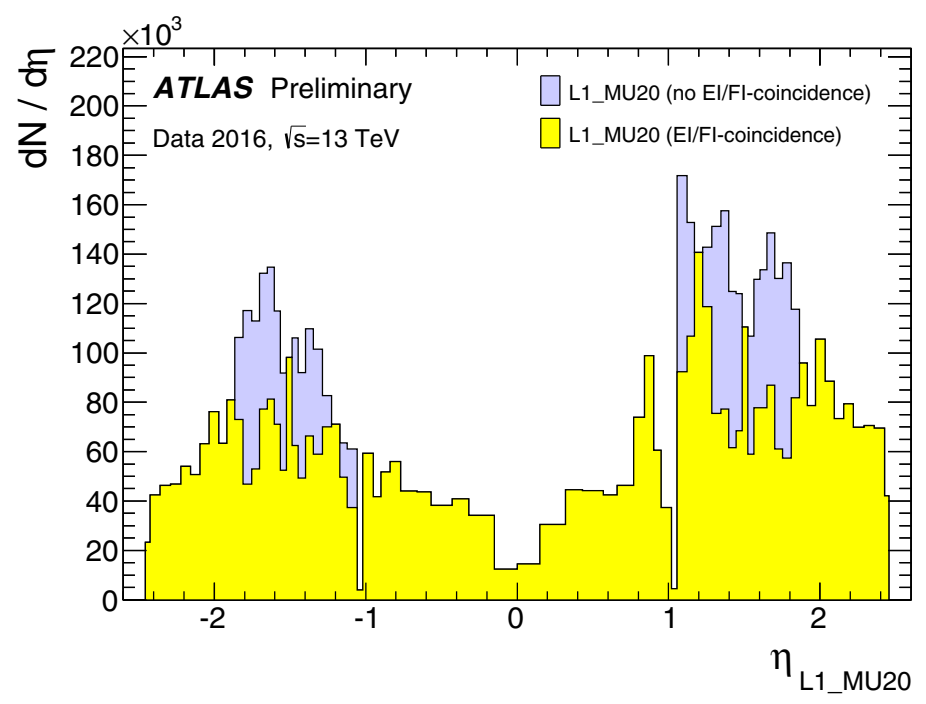

Figure 2. The pseudorapidity $(\eta)$ distributions of the Level 1 muon trigger with a muon $p_{\mathrm{T}}$ threshold of $20 \mathrm{GeV}$ (L1_MU20) with and without the additional coincidence based on the small-wheel TGCs in the FI and the EI chambers [3].

\section{Improvement of the High Level muon trigger for 2018}

The High Level muon trigger in Run 2 is designed to have a two-step approach: a fast and a precise muon reconstruction. In the fast reconstruction, the muon $p_{\mathrm{T}}$ is measured by using the hit information provided by the MDTs and CSCs with fast tracking provided by the ID. If the muons satisfy the requirements in the fast reconstruction, they proceed to the precise muon reconstruction. In the precise reconstruction, the muon $p_{\mathrm{T}}$ is measured by using algorithms close to offline muon reconstruction [2].

An efficiency drop was observed in the isolated muon trigger with a $p_{\mathrm{T}}$ threshold of $26 \mathrm{GeV}$ (mu26_ivarmedium), which has been used as one of the primary triggers. The isolation requirement imposes an upper limit on the momentum fraction of additional tracks in a cone of $\Delta R=\sqrt{(\Delta \phi)^{2}+(\Delta \eta)^{2}}<\Delta R_{\text {cut }}$ around the muon relative to the muon candidate's momentum [2]. In 2017, the trigger efficiency of mu26_ivarmedium dropped by about $4 \%$ for average pileup of 60 . This is due to a cone width requirement in $\mathrm{z}$ direction, i.e. $\Delta z$, which is the difference between the longitudinal impact parameters $z_{0}$ (Figure 3 ) of the track and the muon. A previous $\Delta z$ requirement, $\Delta z<6 \mathrm{~mm}$, was too wide for requiring the isolation, because it picked up other tracks from different vertices (i.e. pileup tracks etc), and then discarded even isolated muons in the high pileup conditions.

The $\Delta z$ requirement was optimised, and the trigger efficiency of mu26_ivarmedium was recovered. Table 1 shows the rates and efficiencies of mu26_ivarmedium for three $\Delta z$ requirements. As shown in Table 1, the trigger efficiency for $\Delta z<2 \mathrm{~mm}$ is recovered by $3 \%$ with a small rate increase due to muons with the related momentum fraction close to the threshold, compared to the previous $\Delta z<6 \mathrm{~mm}$ requirement. Since the rate increase was acceptably low, the $\Delta z$ requirement $\Delta z<2 \mathrm{~mm}$ has been used for the 2018 data taking. 

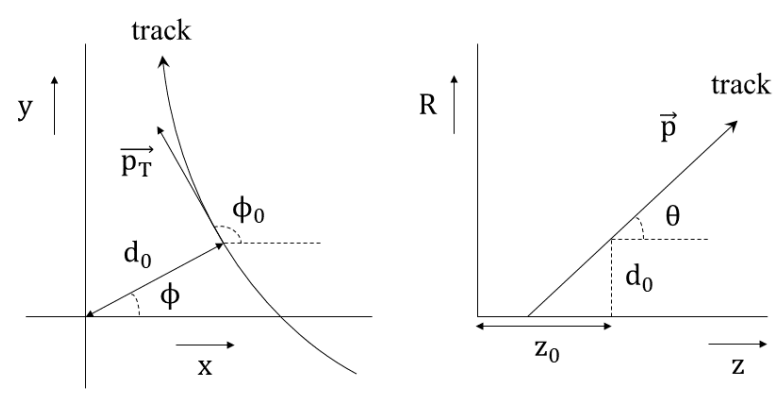

Figure 3. Illustration of the impact parameters of a track in the transverse plan (left) and RZplane (right). The transverse impact parameter $\mathrm{d}_{0}$ is defined as the distance of closest approach to the beam line ( $\mathrm{z}$-axis). The longitudinal impact parameter $\mathrm{z}_{0}$ is defined as the value of $\mathrm{z}$ of the point on the track that determines $d_{0}$. A more detailed description of the impact parameters can be found in Ref. [4].

Table 1. The rate and efficiency of the isolated muon trigger with a $p_{\mathrm{T}}$ threshold of $26 \mathrm{GeV}$ (mu26_ivarmedium) for three $\Delta z$ requirements.

\begin{tabular}{cccc}
\hline Requirement of $\Delta z$ & Rate [Hz] & $\begin{array}{c}\text { Efficiency } \\
\text { at a pileup of } 60\end{array}$ & $\begin{array}{c}\text { Efficiency } \\
\text { at a pileup of } 80\end{array}$ \\
\hline$\Delta z<6 \mathrm{~mm}$ & 223 & $96 \%$ & $91 \%$ \\
$\Delta z<3 \mathrm{~mm}$ & 232 & $98 \%$ & $95 \%$ \\
$\Delta z<2 \mathrm{~mm}$ & 239 & $99 \%$ & $97 \%$ \\
\hline
\end{tabular}

\section{Efficiency measurement in the 2018 data}

The efficiency of the muon trigger is evaluated with a tag-and-probe method using offline selected $Z \rightarrow \mu \mu$ events [5]. For the selection of the $Z$-boson sample, invariant mass of a pair of oppositely charged muons is required to be consistent with the mass of $Z$-boson within $10 \mathrm{GeV}$. If one of the two muons is reconstructed fulfilling the medium identification criteria [5], is matched to a trigger muon, and has $p_{\mathrm{T}}>25 \mathrm{GeV}$, it is a candidate for the tag muon. The other muon is a candidate for the corresponding probe muon. The muon trigger efficiency is defined as the fraction of prove muons as

$$
\text { Efficiency }=\frac{\text { Number of probe muons matched to a trigger muon }}{\text { Number of probe muons }} .
$$

In Eq. (1), the effect of background contribution was found to be negligible.

Figure 4 shows the absolute efficiency of L1_MU20, the absolute efficiency of the OR of mu26_ivarmedium and the High Level muon trigger with a $p_{\mathrm{T}}$ threshold of $50 \mathrm{GeV}$ (mu50) and the relative efficiency of the High Level muon trigger to the Level 1 muon trigger as a function of the offline muon $p_{\mathrm{T}}$. The trigger efficiencies are evaluated separately in the barrel and endcap regions, since the MS features different technologies and has different geometrical acceptance in each region. The muon trigger has high efficiencies for $p_{\mathrm{T}}$ greater than the thresholds. 

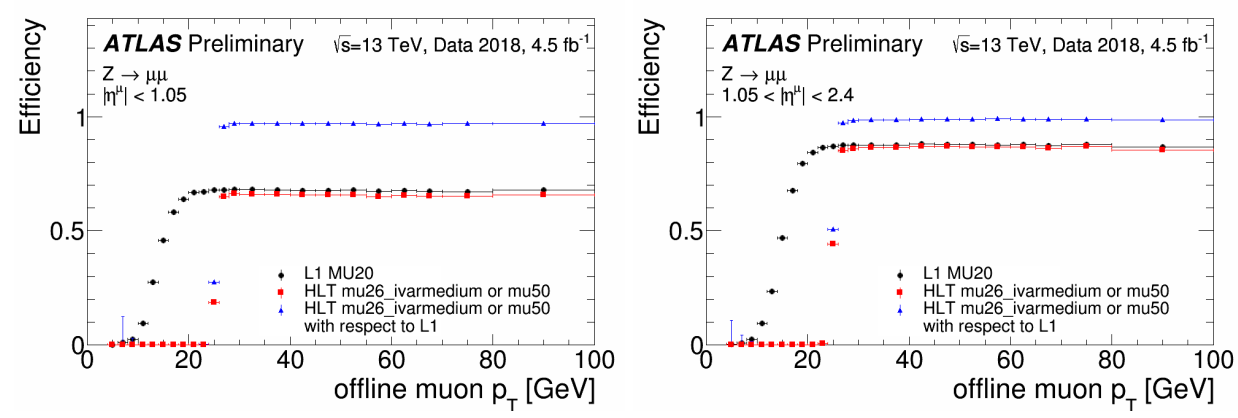

Figure 4. Absolute efficiency of L1_MU20 (black dots), absolute efficiency of the OR of mu26_ivarmedium and mu50 (red squares), and the efficiency of the OR of mu26_ivarmedium and mu50 relative to L1_MU20 (blue triangles) as a function of $p_{\mathrm{T}}$ of offline muon candidates. The plots are shown for barrel (left) and endcap (right) [3].

\section{Conclusion}

The muon trigger system is essential for the physics program of the ATLAS experiment. For Run 2 data taking, several improvements were implemented in the muon trigger to cope with the high instantaneous luminosity and pileup conditions. At the Level 1 muon trigger, an additional coincidence of TGCs with the small-wheel TGCs, the FI and EI chambers, has been implemented. It reduces the trigger rate by about $20 \%$ in the region $1.05<|\eta|<2.0$. At the High Level muon trigger, the trigger efficiency of isolated muon triggers is recovered by $3 \%$ by optimizing the $\Delta z$ requirement. In 2018 data taking, a high efficiency of the muon trigger has been validated by a tag-and-probe method using $Z \rightarrow \mu \mu$ events. The ATLAS muon trigger has excellent performance and is operated smoothly in 2018.

\section{References}

[1] ATLAS Collaboration, The ATLAS Experiment at the CERN Large Hadron Collider, 2008 JINST 3 S08003

[2] ATLAS Collaboration, Performance of the ATLAS muon trigger in pp collisions at $\sqrt{s}=8 \mathrm{TeV}$, Eur. Phys. J. C (2015) 75:120

[3] ATLAS Collaboration, Muon Trigger Public Results https://twiki.cern.ch/twiki/bin/view/AtlasPublic/MuonTriggerPublicResults

[4] ATLAS Collaboration, Expected performance of the ATLAS experiment: Detector, Trigger and Physics, Volume 1, CERN-OPEN-2008-020, December, 2008.

[5] ATLAS Collaboration, Muon reconstruction performance of the ATLAS detector in proton-proton collision data at $\sqrt{s}=13 \mathrm{TeV}$, Eur. Phys. J. C (2016) 76:292 